\title{
Modelo Descritivo do Fluxo de Calor em Soldagem a Arco Visando o Conceito de Calor Imposto Efetivo
}

\section{(Descriptive Model of the Heat Flow in Arc Welding Targeting the Concept of Effective Heat Input)}

\author{
Américo Scotti ${ }^{1}$, Ruham Pablo Reis ${ }^{l}$, Olga Liskevych ${ }^{1}$ \\ ${ }^{1}$ Centro para Pesquisa e Desenvolvimento de Processos de Soldagem (Laprosolda), Universidade Federal de Uberlândia, \\ Uberlândia,Brasil (ascotti@ufu.br)
}

\begin{abstract}
Resumo
O objetivo do presente trabalho é propor um modelo global para descrever o fluxo de calor em soldagem a arco, visando o entendimento dos fenômenos relacionados com o fluxo de calor. A tentativa é suprir uma lacuna do conhecimento gerado entre a aplicação de modelos analíticos simplificados (não totalmente realistas) e de modelos numéricos (que mascaram a visão fisica dos fenômenos). Inicia-se propondo uma descrição mais física para a razão da queda de tensão num arco, baseada na geração e perda de calor para se manter o equilíbrio da desionização e reionização, em contraste com a abordagem em que o arco é comparável a um resistor elétrico. Em seguida, se propõe um modelo descritivo da subsistência térmica de um arco, para qualificar as formas de transferência de calor do arco para a chapa. Um segundo modelo descritivo é então proposto para explicar como o calor entra, difunde e sai da chapa durante a soldagem. Finalmente, é discutido como a aplicação destes dois modelos de forma global permite entender que nem todo o fluxo de calor do arco para a chapa vai afetar a velocidade de resfriamento nas regiões do material aquecidas. Culmina-se com a proposta do emprego do termo Calor Imposto Efetivo como meio de evidenciar o parâmetro de calor de importância a ser determinado em soldagem.
\end{abstract}

Palavras-chave: Calor imposto, fluxo de calor em soldagem, modelos.

Abstract: The objective of the present paper is to propose a global model for describing heat flow in arc welding, aiming the understanding of the phenomena involved. The attempt is to fill out a gap of knowledge which has been generated between the application of simplified analytical models (not totally realistic) and numerical models (that disguises the physics of the phenomena). Initially, a physic-oriented description of the reasons for welding arc voltage drop is presented. This description is based on the heat generation and its losses to maintain the balance between deionization and reionization, in contrast to the approach that the arc is comparable to an electrical resistor. Following, a descriptive model of the thermal subsistence of the arc is proposed to describe the heat transference from the arc to the plate. Then, a second descriptive model is proposed to explain how the heat enters, diffuses and leaves the workpiece during welding. Sequentially, it is discussed how the application of these two models in a global way allows to understand that not all the heat flow from the arc to the plate will affect the cooling rate of the heated parts. A proposal for applying the term Effective Heat Input to mean the heat parameter which is important to be determined in welding is put forward.

Key-words: Heat input, heat flow in welding, models

\section{Introdução}

Para quantificar ou qualificar os efeitos térmicos na soldagem a arco, encontram-se na literatura dois termos frequentemente usados. O primeiro é o calor imposto (correlacionado com o rendimento térmico) e o segundo, menos utilizado, é o calor de fusão, ou suas derivações, como calor efetivo de fusão (correlacionado com o rendimento de fusão). A norma da Sociedade Americana de Soldagem [1] define o calor imposto como a energia entregue para a peça. Este termo é muito aplicado, até em normas [2], para correlacionar os parâmetros de soldagem (energia de soldagem) com as alterações metalúrgicas e respectivas propriedades mecânicas decorrentes. $\mathrm{O}$ calor de

(Recebido em 18/05/2012; Texto final em 30/05/2012). fusão, por outro lado, é relacionado como a parcela do calor imposto na chapa que é efetivamente utilizado para fundir o material [3] (por isto algumas vezes relacionado como calor efetivo de fusão [4]), sendo de extrema importância do ponto de vista da capacidade de produção de um dado processo, pois é correlacionado com a eficiência do calor para formar um cordão de solda.

Entretanto, muitas vezes esses termos são usados de formas muito simplistas na literatura (apenas tomando como base suas definições e não o significado físico dos mesmos), impedindo que os tecnologistas e estudantes possam claramente entender como o fluxo de calor se desenvolve no ambiente de um arco de soldagem e dentro da peça a ser soldada. Só com este entendimento que se poderia esperar uma visão mais precisa dos fenômenos inerentes aos efeitos térmicos, principalmente quanto à influência das condições de contorno sobre o comportamento desse fluxo de calor. Da literatura corrente e grupos de discussão se depreende que para os usuários de soldagem o rendimento 
térmico é dependente do processo de soldagem. Entretanto restam algumas lacunas que não parecem estar esclarecidas quanto ao papel da composição do gás de proteção ou até mesmo dos parâmetros de soldagem. Outras lacunas se estendem à falta de clareza sobre o fato do calor imposto na chapa se comportar de forma diferente em função não só da espessura ou condutividade térmica da chapa, mas também do comprimento de arco, energia de soldagem e até do formato da poça fundida. Reis et al. [4], por exemplo, estudando a influência do material no rendimento de fusão, demostraram que o rendimento bruto de fusão depende significativamente das propriedades do material de base e tende a ser muito baixo para o processo de soldagem TIG CA, mas aumenta com o aumento do nível de corrente de soldagem utilizado. Entre os materiais estudados (aço ao carbono, aço inoxidável e alumínio), o alumínio apresentou o menor rendimento bruto de fusão, o que faz com que boa parte do calor imposto pelo processo de soldagem tenda somente a aquecer o material ao invés de fundi-lo. No caso da soldagem de aço inoxidável, os autores concluíram a ocorrência de fenômeno inverso.

Mesmo o significado real de calor imposto parece não ser muito discutido e esclarecido. Na maioria dos casos, o conceito de calor imposto subentende apenas a quantidade de calor transferido do arco para a peça, medida, por exemplo, através da calorimetria. Mas não é óbvio para todos se todo o calor transferido para a peça age sobre as alterações metalúrgicas. Pode-se imaginar que sempre existirá uma parcela de calor entregue para a peça que sai pela superfície da mesma antes de se difundir para dentro da mesma. Assim como que o calor usado para a fusão do metal em grande parte é difundido para a chapa, o que impede afirmar que um alto rendimento de fusão signifique pouco calor difundido para a chapa (menos distorções, etc.).

Mas na tradição de uso da soldagem, simplesmente assume-se que todo o calor que entra na peça se difunde pela mesma, como quando se aplica modelos baseados na solução analítica simplificada de Rosenthal [5,6]. Com certeza, os modelos numéricos mais recentes [7,8], com alta complexidade, conseguem prever perdas de calor já na chapa para o ambiente por radiação e convecção, por isto com maior aproximação da realidade. Mas os mesmos são pouco usados, talvez até por não transmitir para o usuário de soldagem o entendimento físico do fenômeno. Por isso, os engenheiros de soldagem continuam aplicando fórmulas simplificadas (por exemplo, cálculos online da Sociedade dos Engenheiros de Soldagem do Japão [9]) para calcular velocidades de resfriamento, temperaturas de préaquecimento e prevenir trincas a frio, por exemplo. Estes métodos fornecem certa segurança ao usuário, mas nem sempre refletem os fenômenos que realmente acontecem durante a soldagem. Como consequência, os usuários não conseguem identificar o grau de conservadorismo que se aplica nos resultados.

Mas achar uma forma intermediária entre os modelos numéricos para fluxo de calor em soldagem (mais realistas) e os desenvolvidos pela aplicação de equações simplificadas para solução analítica do problema (menos complexos), de tal forma a permitir aos usuários um entendimento qualitativo dos princípios físicos dos fenômenos envolvidos, passa a ser um desafio para pesquisadores e educadores na área da soldagem.
Alguns autores apresentam modelos descritivos para a perda de calor pelo arco, por exemplo, Tanaka e Lowke [10]. Modelos descritivos da difusão de calor na chapa não são comuns na literatura, a não ser de forma bem básica [11]. Mas um modelo descritivo mais abrangente, que demonstre o fluxo de calor desde sua geração no eletrodo e arco até sua difusão dentro da peça não foi encontrado.

Assim, o objetivo deste trabalho é propor modelos descritivos dos fenômenos que governam o fluxo do calor na soldagem, tanto quando da geração do calor no arco como desse calor aportando na chapa. Espera-se, com isto, facilitar o entendimento sobre os efeitos do fluxo de calor em soldagem e, com isto, justificar a proposição neste trabalho de outra terminologia, a saber, Calor Imposto Efetivo, que configura o segundo objetivo deste trabalho.

\section{Desenvolvimento De Um Modelo Descritivo Global Para O Fluxo De Calor Em Soldagem A Arco}

$\mathrm{Na}$ elaboração de um modelo mais abrangente (global) para o fluxo do calor na soldagem a arco, a proposta foi fazêlo em duas partes, a primeira descritivamente em relação à subsistência do arco, incluindo perdas e ganhos no balanço de energia do arco, e a segunda parte referente à descrição do fluxo de calor imposto dentro da chapa, incluindo a forma de recebimento deste calor e a das perdas de calor pelas superfícies da poça fundida e da chapa.

\subsection{Modelo Descritivo da Subsistência Térmica de um Arco Elétrico de Soldagem}

Na literatura corrente, é comum se referenciar um arco como uma resistência elétrica [12], ou seja, o espaço de separação entre o eletrodo e a peça comporta-se como um resistor. Entretanto, para fins do modelo proposto, a existência do arco é controlada pelas reações acontecidas nos acoplamentos do arco (anódico e catódico) e pelos processos de desionização e reionização na coluna de plasma, os quais ocorrem simultaneamente e constantemente durante a soldagem.

Assim, a primeira fonte para quedas de tensão num arco, inclusive a mais significantes (ver, por exemplo, dados de Scotti et al. [13]) são as devidas às reações físicas (emissão e recepção de elétrons) que acontecem nos acoplamentos arco-eletrodo e arco-peça. Não se trata de uma resistência ôhmica por ser uma superfície de contato de pequena espessura (estimada em $10^{-6}$ $\mathrm{m}$ ), mas de altíssima demanda por potencial elétrico (estimado em $10^{-7}$ a $10^{-9} \mathrm{~V} / \mathrm{m}$ ), não para controlar a passagem de elétrons, mas para permitir a emissão e recepção de elétrons.

Quanto a coluna de plasma, a desionização da coluna de plasma é devida à redução da energia dos átomos, a qual advém em consequência de perdas de calor. A reionização ocorre como resultado de choques inelásticos entre elétrons se movimentando (do polo negativo para o positivo) e partículas não ionizadas (incluindo as desionizadas) ao longo do arco. Os choques transferem energia para as partículas, permitindo à matéria mudar da temperatura ambiente para o estado ionizado. Quanto maior a energia requerida pelo átomo para se tornar ionizado (a 
qual depende de algumas características da matéria em questão, tais como o potencial de ionização, calor específico do volume de matéria no qual os átomos estão, etc.), maior é a diferença de potencial (DDP) demandada, popularmente chamada de tensão requerida.

Além disto, a capacidade de troca do calor do meio que forma o arco também influencia a tensão requerida, uma vez que maiores perdas resultam em mais átomos desionizados (que precisam de reionização) e, consequentemente, maior tensão é exigida para manter o arco à mesma corrente. Quando o comprimento do arco é aumentado por qualquer razão, sua área superficial aumenta e, consequentemente, as perdas de calor (trocas de calor) aumentam. Como visto, considerar o arco como um condutor sólido (resistor), como no sentido geral, pode ser uma abordagem prática, mas imprecisa. Num condutor sólido, a resistência elétrica é ligada à dificuldade de movimento de elétrons através de sua estrutura (quanto maior a área da seção transversal do condutor, menor a DDP). Em meios plasmáticos (arco), por outro lado, ela parece estar relacionada principalmente à necessidade de reionizações (quanto maior a área da seção transversal do condutor, maior a DDP). Assim, o aumento de tensão (DDP) à medida que o arco é alongado seria devido à necessidade de aumentar seu nível de reionização para compensar um aumento do processo de desionização.

É importante mencionar que não existe uma relação direta entre as DDP na coluna de um dado arco (mesmo comprimento e mesmo gás de proteção) e nos acoplamentos do mesmo. Por exemplo, quanto menor o número de elétrons se movimentando ao longo do arco (correntes mais baixas), maior é a DDP requerida, como consequência de um menor número de choques para reionizar um mesmo volume de plasma (os elétrons precisam de maior energia), superando à redução das parcelas de calor oriundas das zonas de acoplamento anódica e catódica, as quais diminuem para menores correntes. Com o aumento progressivo da corrente (maior número de elétrons se movimentando), o número de choques aumenta e a DDP requerida é menor. Como resultado, a tensão do arco como um todo decresce, mesmo que a parcela do calor dos acoplamentos aumente. Mas este comportamento tem um limite, que seria o valor mínimo de tensão (ponto de inflexão da característica estática de um arco). A partir de um valor, o consequente aumento da corrente (número de elétrons) passa a demandar também maior DDP, tanto pelo aumento cada vez maior do calor gerado nos acoplamentos do arco, mas também devido ao aumento do volume do arco (capacidade maior de ionizar o meio), que passa, por outro lado, a ter maior área para troca de calor.

Fazer um diferencial entre um arco ser tratado como uma resistência elétrica e ser tratado como um processo de ionização e desionização é fundamental para a concepção do modelo proposto neste artigo. Neste contexto, para descrever o balanço térmico para subsistência de um arco, considerou-se um arco com eletrodo não consumível (caso típico da soldagem TIG), abordando apenas as entradas e saídas de calor na coluna de plasma, as quais ocorrem durante a manutenção da existência do arco. A Figura 1 apresenta a proposta de um esquema para o balanço de energia no ambiente da coluna de plasma de um arco

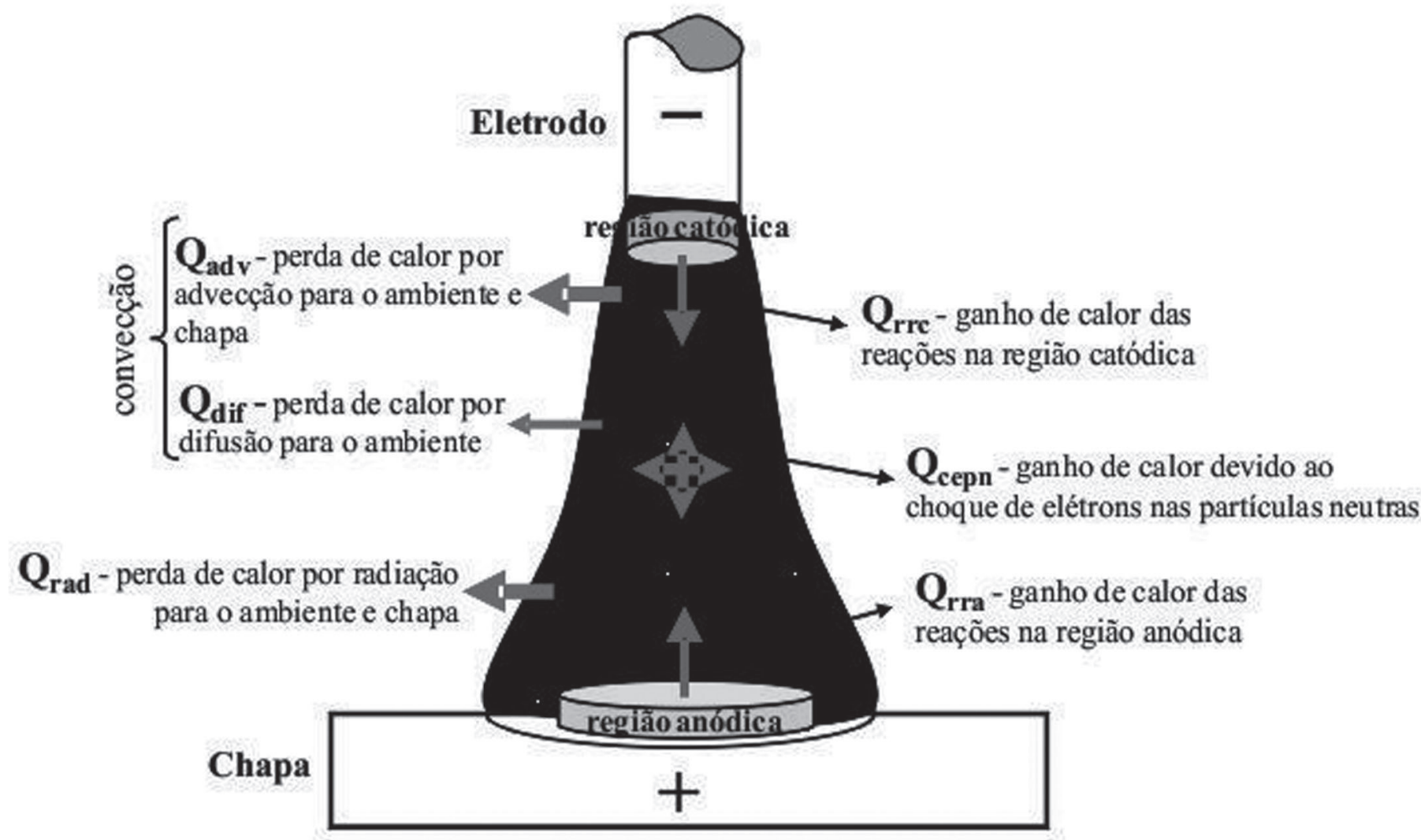

Figura 1: Esquema do balanço de calor na coluna de plasma de um arco TIG 
TIG. A coluna de plasma (arco) existe se o calor total "gerado" e entrando na coluna de plasma se iguala ao calor que deixa esta região do arco (Equação 1). A perda de calor na coluna de plasma ocorre por advecção, difusão e radiação. Entretanto, é comum encontrar referências à convecção, condução e radiação, terminologias que seriam, até certo ponto, incorretas, já que convecção acontece por advecção de calor (devido ao movimento de gás, natural ou forçada) e difusão de calor (também chamada de condução). Como se poderia esperar uma baixa transferência de calor por difusão no plasma, seria mais correto dizer que a perda de calor na coluna de plasma acontece principalmente por advecção e radiação.

$\mathrm{Q}_{\mathrm{rrc}}+\mathrm{Q}_{\text {cepn }}+\mathrm{Q}_{\mathrm{rra}}=\mathrm{Q}_{\mathrm{adv}}+\mathrm{Q}_{\mathrm{dif}}+\mathrm{Q}_{\mathrm{rad}}$

Eq. 1

O termo advecção aqui engloba a perda de calor devido ao fluxo de gás de proteção (existe uma diferença de velocidade entre o fluxo de gás e o jato de plasma) e a perda de calor devido ao movimento de matéria dentro do arco (causado pelo jato de plasma). Uma parte do calor perdido por advecção vai para a chapa. Em termos de contribuição para as perdas de calor na coluna de plasma, como citado por Richardson [14] e Allum [15], a perda calor por convecção é mais significativa. Entretanto, existem controvérsias a este respeito. Tanaka e Lowke [10], por exemplo, fazem menção a radiação como o mecanismo de perda de calor predominante na coluna de plasma.

A partir das considerações acima, é possível dizer que a corrente de soldagem influencia o calor "gerado" e entrando na coluna de plasma. É razoável pensar dessa forma, já que o calor produzido na zona catódica representa a maior parte do calor no arco (maiores temperaturas perto do eletrodo) e grande parte deste calor é transferido por convecção e radiação para a coluna de plasma. Em relação ao comprimento do arco, este fator influencia os termos de saída de calor, já que mudanças no comprimento do arco implicam em mudanças na área superficial do mesmo. Como qualquer perda de calor dependente da área superficial, qualquer aumento no comprimento do arco implica em um aumento da perda de calor por convecção e radiação na coluna de plasma.

Também é importante mencionar que apesar de calor ser gerado também ao longo do eletrodo, aí sim por efeito Joule (resistor), este calor não é transferido para o arco, pelo contrario, retira calor. Por isto não é considerado como entrada no balanço térmico do modelo representado pela Figura 1.

\subsection{Modelo Descritivo do Fluxo de Calor no Material Soldado por Arco Elétrico}

Partindo para a complementação, considera-se agora a chapa e o calor transferido (incluindo a transferência metálica, caso de típico de processos como o $\mathrm{MIG} / \mathrm{MAG}$ e suas derivações) e extraído do material metálico. Os autores propõem, neste sentido, o modelo apresentado esquematicamente na Figura 2. O fluxo de calor entra na peça a partir da energia de soldagem (potência elétrica do arco por unidade do comprimento do cordão, $\mathrm{E}_{\mathrm{s}}$ ), que é a força governante do sistema. A potência elétrica consumida para manter o arco aberto ao longo do processo de soldagem da chapa é determinada a partir das potências instantâneas $\left(\mathrm{P}_{\mathrm{i}}\right)$, como é definido pela Eq. 2, onde $\mathrm{V}_{\mathrm{s}}$ representa a velocidade de soldagem, $I_{i}$ a corrente instantânea, $U_{i}$ a tensão instantânea e $n$ o número das amostras de medição discreta dos sinais elétricos.

$E_{s}=\frac{P_{i}}{V_{s}}=\frac{\sum_{i=1}^{n} I_{i} U_{i}}{n / V_{s}}$

Baseando no raciocínio dos autores do presente artigo, a transferência de calor para a peça acontece principalmente através de três meios. O primeiro acontece na conexão arco-peça (o calor gerado na mancha anódica ou catódica), onde o calor é transferido por condução para a chapa formando a poça de fusão. Quanto maior é a corrente, mais calor é transferido para a superfície da chapa. Assim, seria razoável dizer que mais de $80 \%$ de toda tensão do arco é consumida na conexão arco-peça e arco-eletrodo. Scotti et al. [13], trabalhando com curto-circuito e gás de proteção composto por $\mathrm{Ar}+\mathrm{O}_{2}+\mathrm{N}_{2}$, descobriram que as quedas de tensão nas conexões do arco com o ânodo e com o cátodo apresentam quase a queda de tensão total (a coluna do arco apresenta apenas 3,5 a 4\% da tensão total do arco, que inclui também o eletrodo e as gotas em transferência). Lenivkin et al. [16] encontraram queda no cátodo de $13,2 \mathrm{~V}$, no ânodo de 7,25 $\mathrm{V}$ e o um campo elétrico na coluna do arco de $2,9 \mathrm{~V} / \mathrm{mm}$ para soldagem $\mathrm{MIG} / \mathrm{MAG}$ de aço carbono com proteção de $\mathrm{N}_{2}$ (que corresponde a $12,4 \%$ da queda total do arco para arco de $1 \mathrm{~mm}$ e $29,8 \%$ para arco de $3 \mathrm{~mm}$ ). Dessa forma, mesmo que o valor total das quedas de tensão nas conexões seja o predominante na transferência do calor do arco para a peça, mesmo assim seria impossível prever a porcentagem fixa que realmente entra na chapa (já que esta quantidade de calor depende de um grande número de variáveis, inclusive da composição do gás de proteção).

O segundo meio da transferência do calor para a chapa representa o calor carregado por gotas em transferência do eletrodo para a poça de fusão. Soderstrom et al. [17] encontrou esta quantidade do calor como sendo 20 a $30 \%$ da energia total de soldagem (energia do arco, energia usada para aquecer a extensão do eletrodo e perdas), dependendo do material e tipo da transferência metálica. Vale lembrar que uma parcela do calor carregado pelas gotas é perdida para o ambiente devido aos respingos e à evaporação metálica.

O terceiro meio envolve a energia da coluna de plasma que é entregue para a chapa, principalmente na área adjacente à poça de fusão. A radiação da coluna de plasma é um meio de transferir o calor para a chapa, especialmente fora da conexão arco-peça (devido à alta temperatura dentro dessa região, o calor é transferido por condução, como é visto acima). Entretanto, o jato de plasma também carrega energia do plasma (entalpia) para a chapa através da advecção forçada (movimento forçado do gás) e difusão. Portanto, uma parcela do calor da coluna de plasma se perde para o ambiente (diretamente, ou indiretamente), também por radiação (predominantemente) e 


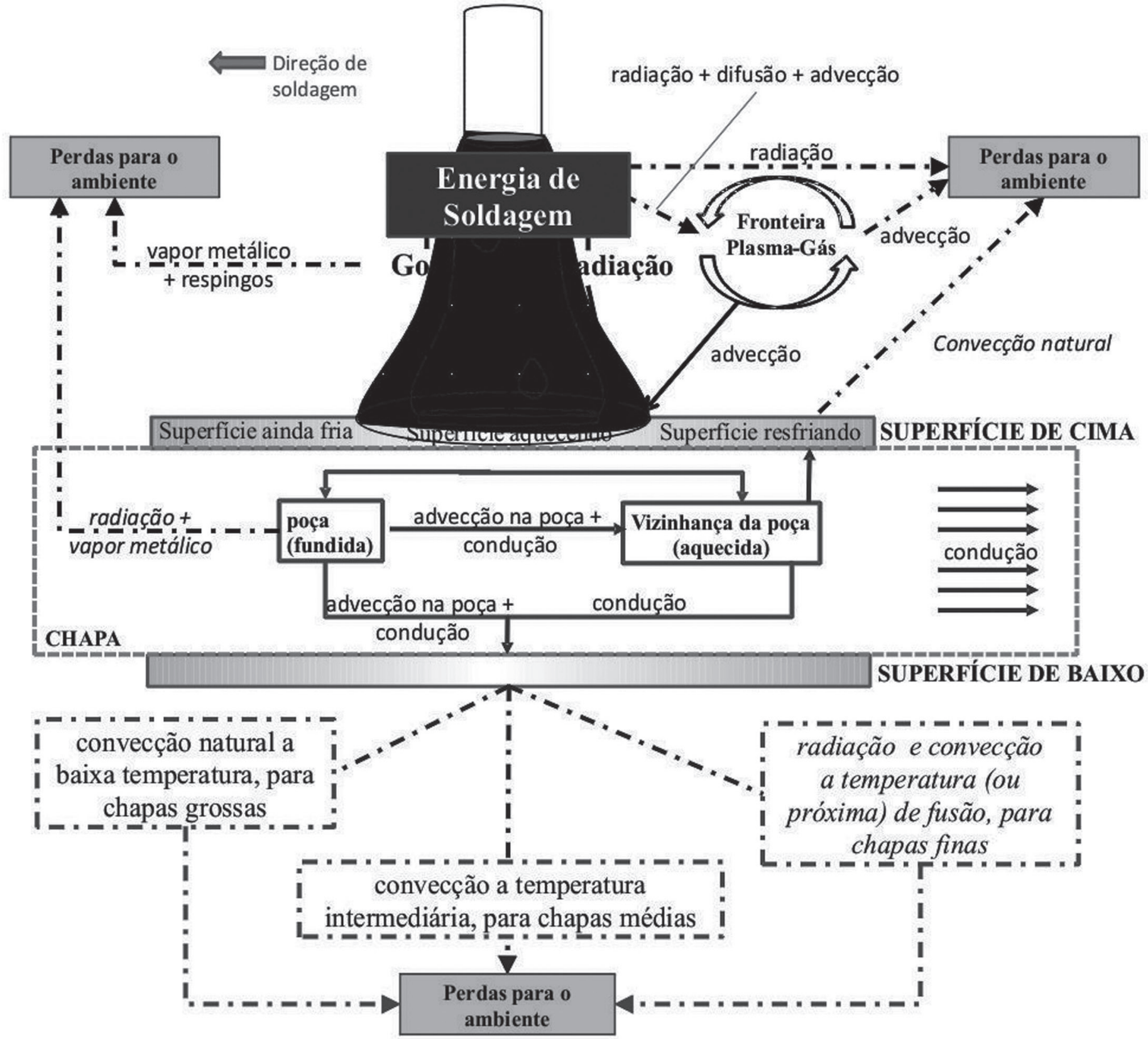

Figura 2 - Apresentação esquemática da entrada e da saída do calor na chapa durante a soldagem (em itálico estão as perdas do calor que não influenciam as velocidades de resfriamento na ZAC)

advecção (há uma diferença entre a velocidade do fluxo do gás e o jato de plasma, aquecendo a fronteira da coluna de plasma gás de proteção). Mas a parcela significante desta perda de calor da coluna de plasma vai para a chapa (não considerada, então, como perda). Isso acontece por advecção, uma vez que a camada correspondente do gás aquecido não ionizado em torno também carregue a energia (entalpia).

Logo que o calor é transferido para a superfície, a maior parte dele vai difundir para dentro da chapa, mas uma parte é liberada de volta para o ambiente por convecção (advecção + difusão dentro da atmosfera) através da superfície ainda quente do cordão já solidificado (volume próximo atrás do arco). Este calor difundido vai tanto fundir uma parte do metal (uma grande quantidade do metal vai fundir-se se, dependendo da concentração e intensidade e coeficiente de difusividade (condutividade térmica) do metal, o calor se acumula localmente antes de difundir pra dentro da chapa) quanto apenas se difundir para dentro da chapa (condução) não afetando diretamente o fenômeno de fusão. O calor que foi usado para fundir o metal depois é eventualmente transferido para a chapa por difusão. Mas uma parte deste calor na poça de fusão é perdida para o ambiente por radiação e convecção natural, através da superfície a alta temperatura do cordão solidificando (exatamente atrás do arco). A outra parcela do calor na poça de fusão também é perdida para o ambiente, tanto antes quanto durante a soldagem, pois tanto as gotas quanto o cordão de solda enquanto líquidos sofrem 
a evaporação e perda de massa (respingos). DuPont e Marder [11] no seu trabalho citam que a evaporação e radiação da poça líquida durante a soldagem de aço foi estimada na ordem de de 30 e $10 \mathrm{~W}$, respectivamente. Mesmo considerando as perdas por convecção, estes autores citam que a perda total do calor durante a soldagem é aproximadamente de $125 \mathrm{~W}$, o que representa por volta de $1 \%$ da potência total do arco (a quantidade é desprezível na calorimetria, de acordo com esses autores).

A maior parte do calor que se difunde para a chapa vai ser distribuída para dentro da chapa (lateralmente), resfriando as partes mais quentes, mais perto do cordão, e aquecendo as partes mais frias, longe do cordão, até que o equilíbrio térmico seja atingido na chapa toda. $\mathrm{O}$ resto das perdas da superfície (convecção natural) não apresenta mais significância quando o aspecto metalúrgico é considerado, pois a temperatura já é abaixo da temperatura de transformação da maioria dos materiais. Por outro lado, a velocidade de resfriamento como uma consequência do processo de condução do calor é o fator governante para transformações metalúrgicas. Portanto, dependendo da espessura da chapa, o calor se difunde através da espessura e atinge a raiz. Dependendo da espessura da chapa e do coeficiente da condutividade térmica, as perdas do calor podem acontecer por três meios: a) perdas por convecção natural a temperatura baixa, se a chapa for grossa o suficiente para não ter a superfície aquecida significativamente (para uma dada energia do arco); b) perdas por radiação e convecção da poça na raiz a alta temperatura e perdas por convecção (raiz do cordão solidificado), se a chapa for fina suficiente para ter o lado oposto aquecido até temperatura de fusão; c) convecção natural a temperatura média, se a espessura da chapa é intermediária.

\section{Conceitos De Calor Imposto Efetivo}

A importância de saber como o calor é entregue para a chapa e como ele é distribuído para dentro da chapa tem o propósito de levantar uma discussão do significado do "calor imposto". A utilização dos modelos propostos permite visualizar como o uso de calor imposto de forma genérica em cálculos baseados em modelos analíticos simplificados leva apenas a aproximações, mas com grandes imprecisões e sem condições de levar em conta a influência de condições de contornos (comprimento de arco, nível de corrente, etc.) sobre o fluxo de calor em soldagem. Esses modelos ajudam ainda a entender melhor os resultados da aplicação de modelos numéricos. Ressalta-se, entretanto, que o modelo descritivo global demonstra o quão complexo é o fenômeno e como seria difícil modelá-lo de forma adequada. Quantificar o calor imposto é uma tarefa muito complexa. Métodos de calorimetria carregam erros intrínsecos. Determinar o calor efetivo de fusão já é mais fácil, mas mesmo assim o valor encontrado não se traduz em informações que possam levar a estimar as transformações metalúrgicas na zona afetada pelo calor (ZAC).

Portanto, os autores propõem um terceiro termo para o jargão de soldagem, o "calor imposto efetivo", para descrever o calor imposto que realmente afeta a velocidade de resfriamento, i.e., o calor interno que se difunde realmente para dentro da chapa através da ZAC do cordão. Como visto no modelo da Figura
2, as perdas marcadas em itálico não influenciam a velocidade de resfriamento, por ocorrerem antes de se difundirem pela ZAC. Isso significa que, para o mesmo calor imposto medido em testes calorimétricos, o calor efetivo pode ser diferente e, consequentemente, também as velocidades de resfriamento nas diferentes partes do metal. O calor imposto efetivo depende do processo de soldagem, da espessura da chapa, dos parâmetros de soldagem, etc. Quanto maior é o calor imposto efetivo, mais lenta é a velocidade de resfriamento na ZAC.

Baseado nos conceitos propostos, outro desafio tecnológico seria o de se encontrar um método de estimação (provavelmente, numérico) não do calor imposto convencional, mas o do calor imposto efetivo, que poderia levar a recomendações e previsões mais realísticas em soldagem.

\section{Conclusões}

Os modelos descritivos propostos permitem de forma qualitativa entender os efeitos do fluxo de calor sobre a chapa soldada e, com isto, justificar a imprecisão da utilização de modelos analíticos simplificados para determinar parâmetros operacionais de soldagem (temperatura de pré-aquecimento, previsão de dureza na ZAC, etc.). Também permitem um melhor entendimento físico do processo, justificando discrepâncias dos resultados de métodos numéricos para cálculo do fluxo de calor em soldagem.

De forma mais específica, as perdas entre a energia de soldagem e o calor imposto não são dependentes apenas do processo, mas de diversos parâmetros do próprio processo, o que não permite o uso de um único valor de rendimento térmico para um dado processo como valor absoluto. O calor que é transferido para a chapa tem origem tanto na conexão do arco com a peça como do próprio plasma (radiação e convecção) e do metal de adição. E nem todo calor que é imposto na peça vai se difundir para dentro da mesma, podendo parte ser transferida para o meio ambiente, o que faz com que a espessura tenha um papel importante na quantidade de calor que se difunde pela chapa. Finalmente, a análise mostra que ao invés de se usar o termo "calor imposto", torna-se mais apropriado se falar em "calor imposto efetivo", por ser a melhor maneira de se correlacionar a energia de soldagem com as transformações metalúrgicas.

\section{Agradecimentos}

Os autores gostariam de agradecer aos órgãos brasileiros de fomento à pesquisa, CNPq e Fapemig, pelo suporte financeiro na forma de bolsas e infraestrutura laboratoriais para a realização de experimentos que levaram às conceituações mostradas neste artigo.

\section{Referências Bibliográficas}

[1] AWS, Standard Welding: terms and Definitions, Standard AWS A3.0:2001, Jul 2001.

[2] MELFI, T., New Code Requirements for Calculating Heat Input, Welding Journal, 89 (6), 2010, pp. 61-65. 
[3] GAO, M., ZENG, X. Y., HU, Q. W, Effects of welding parameters on melting energy of $\mathrm{CO} 2$ laser-GMA hybrid welding, Science and Technology of Welding \& Joining, Volume 11, Number 5, September 2006 , pp. 517-522(6).

[4] REIS, R. P., COSTA, A. L. G., SILVEIRA, F. M., MOTA, P. R., SOUZA, R. J, SCOTTI, A., Influência do tipo de material no rendimento de fusão em soldagem a arco, Soldag.\& Insp., vol.16 no.4, Oct./Dec. 2011.

[5] FUERSCHBACH, P. W. E KNOROVSKY, G. A., A study of melting efficiency in Plasma Arc and Gas Tungsten Arc welding, Welding research, October, 1991, pp. 287-297.

[6] SCHWEDERSKY, M. B. e DUTRA, J. C., Um Estudo da Eficiência Térmica dos Principais Processos de Soldagem a Arco. XXXVII CONSOLDA, Natal-RN, Out. 2011.

[7] MAITI, S. K., WALSH, C. A., BHADESHIA, H. K. D., Finite element simulation of laser spot welding, Science and Technology of Welding \& Joining, Volume 8, Number 5, October 2003 , pp. 377-384.

[8] DENG, D., LIANGB, W., MURAKAWAB, H., Determination of welding deformation in fillet-welded joint by means of numerical simulation and comparison with experimental measurements, Journal of Materials Processing Technology, Volume 183, Issues 2-3, 23 March 2007, pp. 219-225.

[9] JWES, Calculation menu of steel weldability and weld metal property. Em: <http://www-it.jwes.or.jp/weld_simulator/en/ index.jsp>. Acesso em: 08 maio 2012.

[10] TANAKA, M., LOWKE, J. J., Predictions of weld pool profiles using plasma physics. Topical Review. Journal of Physics D: Applied Physics, v. 40, R1-R23, 2007.

[11] DUPONT, J. N. AND MARDER, A. R., Thermal Efficiency of Arc Welding Processes, Weld J, 1995, 74 (12), pp. 406s-416s.

[12] KODAMA, S., IKUNO, Y., ICHIYAMA, Y., BABA, N., Process Modeling of Short-Circuiting GMA Welding and Its Application to Arc Sensor Control, NIPPON STEEL TECHNICAL REPORT No. 95, Jan 2007, UDC 621 . 791.75 $: 681.3$.

[13] SCOTTI, A., PONOMAREV, V. \& COSTA, A. V., A methodology for voltage drop determination in GMA welding: arcs with short-circuiting metal transfer, Eur. Phys. J. Appl. Phys., EDP Sciences, 34, 2006, pp. 231-236.

[14] RICHARDSON, I. M. Introduction to Arc Physics. M.Sc. course hand-out, Cranfield Institute of Technology, SIMS, 1989. [15] ALLUM, C. J. Power dissipation in the column of a TIG welding arc. Journal of Physics D: Applied Physics, v. 16 (11), pp. 2149-2165, 1983.

[16] LENIVKIN, V. A., DIURGEROV, N. G., SAGIROV, K. H. N., Technological Properties of the MIG/MAG Welding Arc, 1st ed., Mashinostroenie, Moscow, 1989 (in Russian).

[17] SODERSTROM, E. J., SCOTT, K. M., MENDEZ, P. F., Calorimetric Measurement of Droplet Temperature in GMAW, Welding Journal, AWS, Apr 2011, 90 (1), pp. 1s-8s. 